\title{
$N$-acetyl-l-cysteine controls osteoclastogenesis through regulating Th17 differentiation and RANKL in rheumatoid arthritis
}

\author{
Hae-Rim Kim ${ }^{1,}$, Kyoung-Woon Kim ${ }^{2,}$, Bo-Mi Kim², Kyung-Ann Lee ${ }^{1}$, and Sang-Heon Lee ${ }^{1}$
}

${ }^{1}$ Division of Rheumatology, Department of Internal Medicine, Konkuk University School of Medicine, Seoul; ${ }^{2}$ Convergent Research Consortium for Immunologic Disease, College of Medicine, Seoul St. Mary's Hospital, The Catholic University of Korea, Seoul, Korea

Received: October 31, 2016 Revised : December 4, 2016 Accepted: December 4, 2016

\section{Correspondence to}

Sang-Heon Lee, M.D.

Division of Rheumatology,

Department of Internal

Medicine, Konkuk University

School of Medicine, 120

Neungdong-ro, Gwangjin-gu,

Seoul 05029, Korea

Tel: +82-2-2030-7541

Fax: +82-2-2030-7748

E-mail: shlee@kuh.ac.kr

*These authors contributed equally to this work.
Background/Aims: This study aimed to determine the regulatory role of $\mathrm{N}$-acetyl-1-cysteine (NAC), an antioxidant, in interleukin 17 (IL-17)-induced osteoclast differentiation in rheumatoid arthritis (RA).

Methods: After RA synovial fibroblasts were stimulated by IL-17, the expression and production of receptor activator of nuclear factor $\kappa$-B ligand (RANKL) was determined by real-time polymerase chain reaction and enzyme-linked immunosorbent assay (ELISA). Osteoclastogenesis was also determined after co-cultures of IL-17-stimulated RA synovial fibroblasts, Th17 cells and various concentrations of NAC with monocytes. After human peripheral $\mathrm{CD}_{4}{ }^{+} \mathrm{T}$ cells were cultured with NAC under Th17 condition, IL-17, interferon $\gamma$, IL-4, Foxp3, RANKL, and IL-2 expression and production was determined by flow cytometry or ELISA.

Results: When RA synovial fibroblasts were stimulated by IL-17, IL-17 stimulated the production of RANKL, and NAC reduced the IL-17-induced RANKL production in a dose-dependent manner. NAC decreased IL-17-activated phosphorylation of mammalian target of rapamycin, c-Jun N-terminal kinase, and inhibitor of $\kappa \mathrm{B}$. When human peripheral blood $\mathrm{CD}_{1} 4^{+}$monocytes were cultured with macrophage colony-stimulating factor and IL-17 or RANKL, osteoclasts were differentiated, and NAC reduced the osteoclastogenesis. After human peripheral $\mathrm{CD}_{4}{ }^{+} \mathrm{T}$ cells were co-cultured with IL-17-pretreated RA synovial fibroblasts or Th17 cells, NAC reduced their osteoclastogenesis. Under Th17 polarizing condition, NAC decreased Th17 cell differentiation and IL-17 and RANKL production.

Conclusions: NAC inhibits the IL-17-induced RANKL production in RA synovial fibroblasts and IL-17-induced osteoclast differentiation. NAC also reduced Th17 polarization. NAC could be a supplementary therapeutic option for inflammatory and bony destructive processes in RA.

Keywords: Acetylcysteine; Arthritis, rheumatoid; Osteogenesis; RANK ligand; Interleukin-17

\section{INTRODUCTION}

Rheumatoid arthritis (RA) is a systemic autoimmune disease characterized by synovial inflammation and subsequent joint destruction [1]. Among the various pathologic events occurring in the affected joints, bone destruction is the most clinically relevant feature because it is related to functional impairment and the 
progression of joint damage among patients with RA in whom the disease is in prolonged remission [2]. Osteoclasts, which are implicated in the development of bony erosions in RA, are specialized bone-resorbing cells regulated by receptor activator of nuclear factor $\kappa-B$ ligand (RANKL) and macrophage colony-stimulating factor (M-CSF) [3].

Among the various immune cells, $\mathrm{CD}_{4}^{+} \mathrm{T}$ cells are known to be involved in the pathogenesis of RA. RA was previously considered to be a purely Th1-mediated disease. This classic paradigm was maintained until 2005, when a distinct lineage of proinflammatory $\mathrm{T}$ helper cells, termed Th17 cells, was identified [4]. Th17 cells are characterized by the production of a proinflammatory cytokine, interleukin 17 (IL-17). Th17 cells and IL-17 significantly contribute to the development and progression of RA [5]. IL-17 induces RANKL expression by synovial fibroblasts and osteoblasts to drive bone erosion indirectly (via metalloproteases), enable activated $\mathrm{T}$ cells to penetrate the extracellular matrix [6], and activate synovial macrophages to secrete tumor necrosis factor $\alpha$ (TNF- $\alpha$ ) and IL-1 $\beta$, two major osteoclastogenic factors [7]. Synovial macrophages can be differentiated into fully functional bone-resorbing osteoclasts, and Th17-induced differentiation of synovial macrophages into osteoclasts represents an important cellular mechanism in bone destruction associated with RA [8].

Increased oxidative stress and elevated production of TNF- $\alpha$, IL-1 $\beta$, and IL- 6 affect bone metabolism in the context of obesity through several mechanisms [9]. Proinflammatory cytokines and oxidative stress are both capable of stimulating osteoclast differentiation and activation [10], and reactive oxygen species (ROS) directly stimulate osteoclast formation and activity, leading to bone resorption [11]. Restoring antioxidant defenses by administration of $\mathrm{N}$-acetyl-1-cysteine (NAC), an antioxidant and glutathione precursor, or ascorbate inhibits RANKL-induced osteoclastogenesis [12] and prevents estrogen deficiency-induced bone loss in mice [13]. In contrast, increasing oxidative stress by depleting glutathione using the specific glutathione synthesis inhibitor 1-buthionine-(S,R)-sulfoximine (BSO) increases osteoclast differentiation and causes substantial bone loss [13]. Furthermore, NAC supplementation in the diet mitigates bone loss, downregulates Wnt signaling, and decreases serum bone-formation markers in an eth- anol-induced oxidative stress mouse model [14]. The benefit of NAC is demonstrated by its ability to prevent the expression of lipopolysaccharide-induced inflammatory mediators (IL-1 $\beta$, IL-6, and IL- 8 ) in phagocytic cells and gingival fibroblasts during the inflammatory process, suggesting a direct link between the production of cytokines and the generation of ROS [15]. As a direct result of these actions, NAC suppresses osteoclast differentiation, which leads to the prevention of bone resorption [12].

In this study, we assessed whether IL-17 played a crucial role in the process of bone destruction in RA through upregulation of RANKL or direct induction of osteoclast differentiation. Additionally, we evaluated whether NAC suppressed IL-17-induced RANKL production and osteoclastogenesis. Our findings provided important insights into the potential applications of this antioxidant as a new therapeutic option for RA-associated joint destruction.

\section{METHODS}

\section{Patients}

Informed consent was obtained from all patients, and the experimental protocol was approved by Institutional Review Board for Human Research, Konkuk University Hospital (KUH1010186). Synovial tissues were isolated from eight RA patients (mean age, $63.4 \pm 4.6$ years; range, 38 to 76 ) undergoing total knee replacement surgery.

\section{Isolation of synovial fibroblasts}

Synovial fibroblasts were isolated by enzymatic digestion of synovial tissues obtained from RA patients undergoing total knee replacement surgery, as described previously $[16,17]$.

\section{Reagents}

Recombinant human RANKL (rhRANKL) and M-CSF were purchased from R\&D Systems (Minneapolis, MN, USA). NAC was obtained from Sigma Chemical Co. (St. Louis, MO, USA). Anti-human IL-17, anti-interferon $\gamma$ (anti-IFN- $\gamma$ ), anti-IL-10, anti-IL-2, anti-IL-1 $\beta$, anti-TNF- $\alpha$, anti-IL-6, and anti-RANKL antibodies were purchased from R\&D Systems. 


\section{Identification of ex vivo peripheral blood mononu- clear cells}

Peripheral blood mononuclear cells (PBMCs) were prepared from heparinized blood by Ficoll-Hypaque (GE Healthcare, Chicago, IL, USA) density-gradient centrifugation.

\section{Induction of Th17 polarizing conditions}

To induce Th17 polarizing conditions, PBMCs were incubated for 48 hours with anti-CD3 $(1 \mu \mathrm{g} / \mathrm{mL}$; BD, Franklin Lakes, NJ, USA), anti-CD28 (1 $\mu \mathrm{g} / \mathrm{mL} ; \mathrm{BD}), \mathrm{IL}-1 \beta$ (20 $\mathrm{ng} / \mathrm{mL})$, IL-6 (20 ng/mL), IL-23 (20 ng/mL), IFN- $\gamma$-neutralizing antibody $(2 \mu \mathrm{g} / \mathrm{mL})$, and IL-4-neutralizing antibody $(2 \mu \mathrm{g} / \mathrm{mL})$. To examine the immunosuppressive effects of NAC, PBMCs were pre-incubated for 1 hour with NAC, and then stimulated as described above.

\section{Expression of RANKL mRNA by real-time poly- merase chain reaction}

Synovial fibroblasts were stimulated with IL-17. For RANKL signal pathway analysis, the fibroblast-like synoviocyte (FLS) were incubated in the presence or absence of NAC for 3 hours before the addition of IL- 17 . After incubation for 72 hours, mRNA was extracted using RNAzol B (Biotex Laboratories, Houston, TX, USA) according to the manufacturer's instructions.

\section{Enzyme-linked immunosorbent assay}

Soluble RANKL, IL-17, IFN- $\gamma$, and IL-2 levels in the culture supernatants from RA-FLS or PBMCs were measured using sandwich enzyme-linked immunosorbent assay according to the manufacturer's instructions.

\section{Western blotting analysis}

The primary antibody to phospho-mammalian target of rapamycin (mTOR), AMP-activated protein kinase (AMPK), Akt, phospho-c-Jun N-terminal kinase (JNK), phospho-extracellular signal-regulated kinase, or phospho-inhibitor of $\kappa B \alpha$ (I $\mathrm{B}-\alpha$, Cell Signaling Technology Inc., Danvers, MA, USA) was diluted 1:1,000 in 0.1\% Tween 20/1x Tris-buffered saline (TTBS), and incubated overnight at $4^{\circ} \mathrm{C}$. The membranes were washed with TTBS, horseradish peroxidase-conjugated secondary antibody was added, and the membranes were incubated for 1 hour at room temperature. After washing with TTBS, the hybridized bands were detected using an ECL detection kit (Amersham Pharmacia, Piscataway, NJ, USA).

\section{Flow cytometric analysis}

Cells were stained with combinations of the following mAbs: CD4-PE/Cy7 and CD25-APC (BD). Cells were washed, fixed, permeabilized, and stained to detect intracellular cytokines with mAbs to IL-17, IFN- $\gamma$, IL-4, and forkhead box $\mathrm{P}_{3}$ (Foxp3, eBioscience, San Diego, CA, USA). Cells were analyzed on a FACS Calibur flow cytometry system (BD).

\section{Osteoclast formation}

As described above, monocytes were added to the IL-17-pretreated FLS with fresh media. Monocytes were co-cultured for 3 weeks in $\alpha$-minimal essential medium and $10 \%$ fetal bovine serum in the presence of $25 \mathrm{ng} / \mathrm{mL}$ recombinant human M-CSF. The addition of rhRANKL protein, prepared as described previously [18], was used as a positive control. On day 21 , tartrate-resistant acid phosphatase (TRAP)-positive cells were identified using a leukocyte acid phosphatase kit according to the manufacturer's protocol [19].

\section{Statistical analysis}

The data are expressed as mean \pm standard error mean (SEM). Statistical analysis was performed using the Mann-Whitney $U$ test for independent samples and the Wilcoxon signed-rank test for related samples. In all analyses, $p<0.05$ was taken to indicate statistical significance.

\section{RESULTS}

NAC reduced IL-17-induced RANKL gene expression and protein production in RA synovial fibroblasts

To confirm the inhibitory effects of NAC in IL-17-induced RANKL expression, RA synovial fibroblasts were pre-incubated with NAC for 3 hours. The synovial fibroblasts were then cultured with various concentrations of IL-17 for 72 hours. Our results showed that IL-17 induced RANKL expression and production. Maximal effects were observed at a concentration of $20 \mathrm{ng} / \mathrm{mL}$; therefore, we used $20 \mathrm{ng} / \mathrm{mL}$ as an optimal dose (data not shown). NAC reduced the IL-17-induced expression 
A
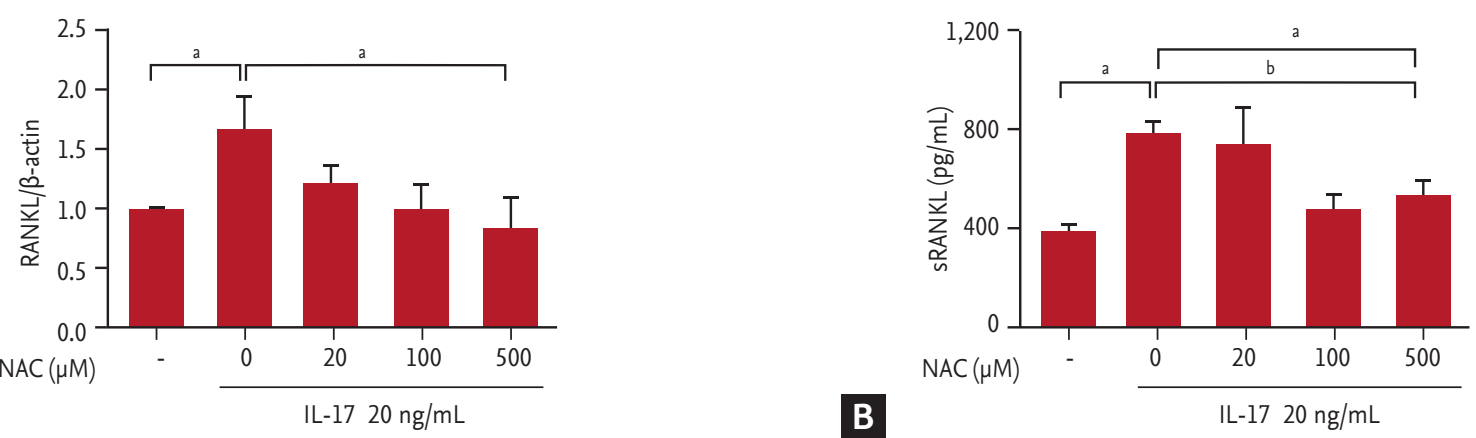

Figure 1. Effect of $\mathrm{N}$-acetyl-l-cysteine (NAC) on receptor activator of nuclear factor $\kappa$-B ligand (RANKL) expression in rheumatoid arthritis (RA) synovial fibroblasts. (A) RA synovial fibroblasts were pretreated with NAC, and then cultured with $20 \mathrm{ng} / \mathrm{mL}$ interleukin 17 (IL-17) for 72 hours. RANKL mRNA level was quantified by real-time polymerase chain reaction. (B) RA synovial fibroblasts were treated with IL-17 in the presence of NAC for 3 hours and the production of soluble receptor activator of nuclear factor $\kappa$-B ligand (sRANKL) was measured by enzyme-linked immunosorbent assay. The data represent the mean \pm SEM of three independent experiments. ${ }^{\mathrm{a}} \mathrm{p}<0.05,{ }^{\mathrm{b}} \mathrm{p}<0.01$.

of RANKL mRNA in a concentration-dependent manner (Fig. 1A). NAC also decreased the production of RANKL by synovial fibroblasts, showing a pattern similar to that observed for mRNA expression (Fig. 1B). NAC did not affect the IL-17-induced production of IL-1 $\beta$, TNF- $\alpha$, and IL-16 (data not shown). The experimental concentrations of NAC had no cytotoxic or proliferative effects on synovial fibroblasts (data not shown).

\section{Signal pathways involved in the inhibitory effects of NAC in RA synovial fibroblasts}

Using RA synovial fibroblasts, we investigated the molecular mechanisms through which NAC modulates IL-17. As shown in Fig. 2, IL-17 enhanced the phosphorylation of mTOR, JNK, and inhibitor of $\kappa \mathrm{B} \alpha$ (IкB- $\alpha$ ), whereas NAC significantly decreased the IL-17-induced phosphorylation of mTOR, JNK, and its downstream

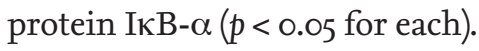

\section{The regulatory effects of NAC in IL-17-induced os- teoclast differentiation}

Peripheral blood $\mathrm{CD}_{1} 4^{+}$monocytes are osteoclast precursors and can differentiate into $\mathrm{TRAP}^{+}$multinucleated osteoclasts in the presence of RANKL and M-CSF, representing a traditional culture system for osteoclastogenesis from osteoclast precursors [20-22]. To evaluate the effects of NAC on IL-17-induced osteoclast differentiation, isolated $\mathrm{CD}_{1} 4^{+}$monocytes from human peripheral blood were cultured with IL-17 and M-CSF in the absence of RANKL. After 21 days of culture, TRAP ${ }^{+}$ multinucleated osteoclasts were differentiated. However, preincubation with NAC significantly decreased IL17-induced osteoclastogenesis in a concentration-dependent manner (Fig. 3A). The mRNA expression of osteoclast markers, such as TRAP, RANK, and calcitonin receptor was also increased with IL-17 stimulation but reduced by NAC treatment (data not shown).

The isolated $\mathrm{CD}_{1} 4^{+}$monocytes were cultured with RANKL and M-CSF. After 21 days of culture, TRAP ${ }^{+}$ multinucleated osteoclasts were differentiated from their precursors in the RANKL and M-CSF culture system. Notably, the osteoclastogenic effect was three times greater than that in the IL-17 and M-CSF culture system. However, preincubation with NAC significantly decreased RANKL-induced osteoclastogenesis (Fig. 3B).

RA-FLS were pretreated with IL-17 and NAC and then were added to a culture system with $\mathrm{CD}_{1} 4^{+}$monocytes and M-CSF. When the monocytes were co-cultured with IL-17-pretreated RA-FLS in the absence of RANKL, $\mathrm{TRAP}^{+}$multinucleated osteoclasts showed greater differentiation than that observed when monocytes were co-cultured with untreated RA-FLS. NAC suppressed the differentiation of osteoclasts induced by IL-17-pretreated FLS (Fig. 3 C). The mRNA expression levels of osteoclast markers, such as TRAP, RANKL, and cathepsin $\mathrm{K}$, were increased in co-culture with IL-17-pretreated FLS; however, NAC blocked this effect (data not shown).

When osteoclast precursors were cultured with $\mathrm{CD}_{4}{ }^{+}$ $\mathrm{T}$ cells, osteoclastogenesis was decreased because of the antiosteoclastogenic effects of IFN- $\gamma$ produced by $\mathrm{CD}_{4}{ }^{+}$ 


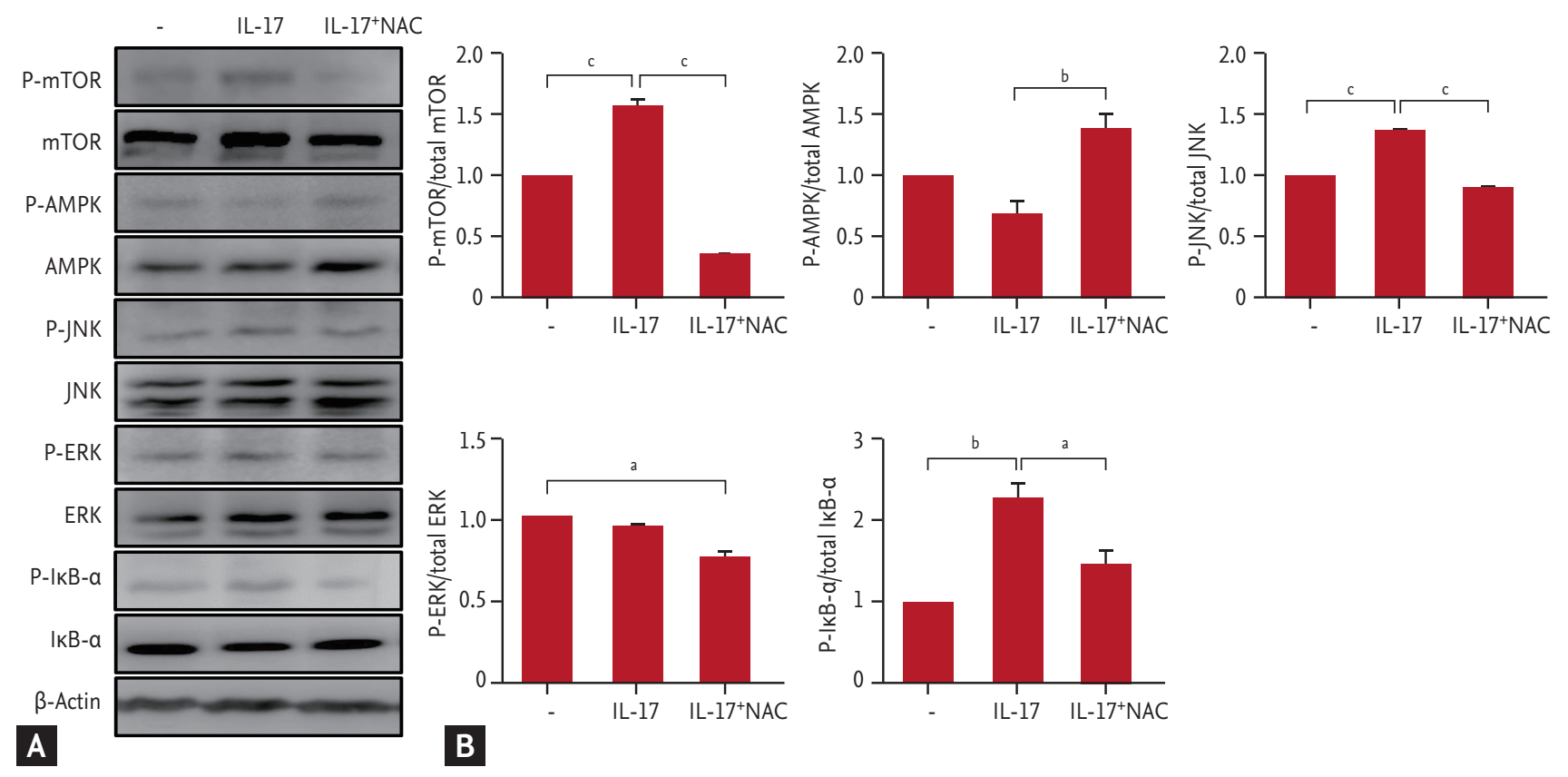

Figure 2. Effects of $\mathrm{N}$-acetyl-1-cysteine (NAC) on the expression of mammalian target of rapamycin (mTOR) and AMP-activated protein kinase (AMPK) proteins in rheumatoid arthritis (RA) synovial fibroblasts. (A) RA synovial fibroblasts were pretreated with NAC, and then cultured with $20 \mathrm{ng} / \mathrm{mL}$ interleukin 17 (IL-17) for 1 hour. RA synovial fibroblasts were stimulated with 20 $\mathrm{ng} / \mathrm{mL}$ IL-17, the phosphorylated forms of target and $\beta$-actin were detected by Western blotting. (B) Data were normalized to $\beta$-actin and reported in relative expression units. The data represent the mean \pm SEM of three independent experiments. P-, phosphorylated; JNK, c-Jun N-terminal kinase; ERK, extracellular signal-regulated kinase; IкB- $\alpha$, inhibitor of $\kappa \mathrm{B} \alpha .{ }^{\mathrm{a}} p<0.05$, ${ }^{b} p<0.01,{ }^{c} p<0.001$.

T cells. However, Th17 cells have high inflammatory and destructive potential and can induce osteoclastogenesis [23]. When isolated monocytes were co-cultured with Th17 cells and M-CSF in the absence of RANKL, TRAP ${ }^{+}$ multinucleated cells showed increased differentiation, albeit without statistical significance. NAC suppressed the differentiation of osteoclasts induced by untreated T cells and Th17 cells (Fig. $3 \mathrm{D}$ ).

\section{Effects of NAC on Th17 cell differentiation}

PBMCs were isolated from healthy individuals and cultured under Th17-polarizing conditions in the presence of NAC (Fig. 4A). NAC significantly reduced the percentage of $\mathrm{IL}_{-1} 7^{+} / \mathrm{CD}_{4}{ }^{+} \mathrm{T}$ cells. However, NAC did not affect the differentiation of the cells into $\mathrm{CD}_{2} 5^{+} \mathrm{Foxp}_{3}{ }^{+} \mathrm{CD}_{4}{ }^{+} \mathrm{T}$ cells, IFN $-\gamma^{+} \mathrm{CD}_{4}{ }^{+} \mathrm{T}$ cells, or IL $-4^{+} \mathrm{CD} 4^{+} \mathrm{T}$ cells (Fig. $4 \mathrm{~B}$ ). NAC also reduced the secretion of IL-17 and RANKL into the cultured medium (Fig. 4C).

\section{DISCUSSION}

In RA, oxidative stress is an important mechanism causing destructive proliferative synovitis [11]. Moreover, overproduction of TNF- $\alpha$ increases ROS production, resulting in inflammation and tissue destruction. RANKL induces the expression of nitric oxide synthase, and NAC attenuates RANKL-induced ROS production and osteoclast differentiation in bone marrow monocyte-macrophage lineage cells [12]. Additionally, osteoclasts are activated by ROS to enhance bone resorption [24]; however, few studies have investigated the role of ROS in the differentiation of macrophages and monocytes into osteoclasts. Sequential processes are involved in osteoclast differentiation and function, including differentiation from osteoclast precursors, cellular fusion to multinucleated osteoclasts, activation of multinucleated osteoclasts, and sustained survival of the osteoclasts $[25,26]$. M-CSF, RANKL, TNF- $\alpha$, IL-17, and IL-1 $\beta$ are the primary molecules involved in osteoclastogenesis, although various other cytokines and chemokines are also 

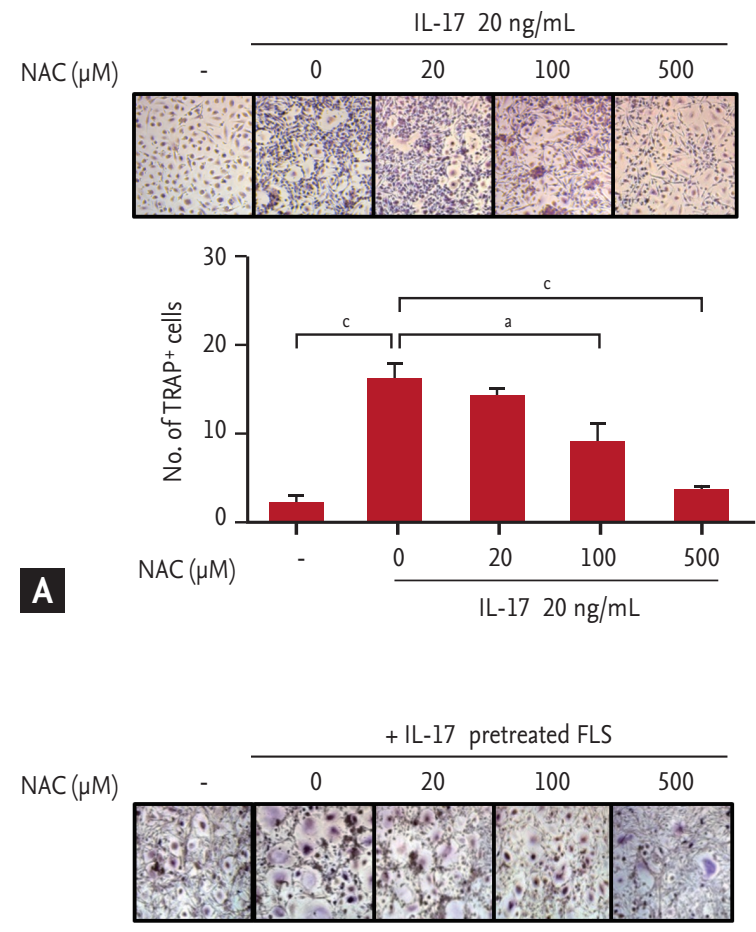

C

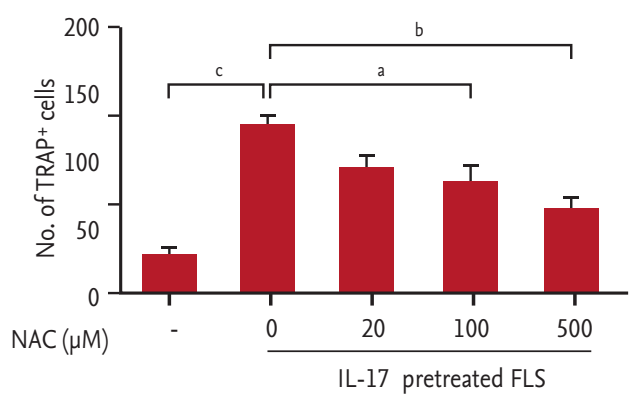

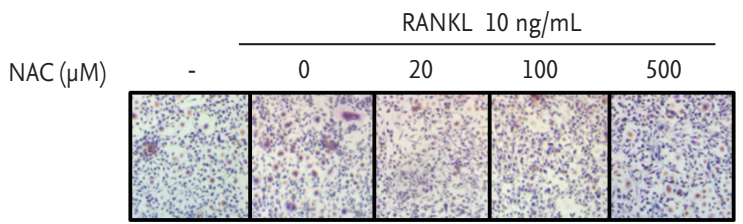
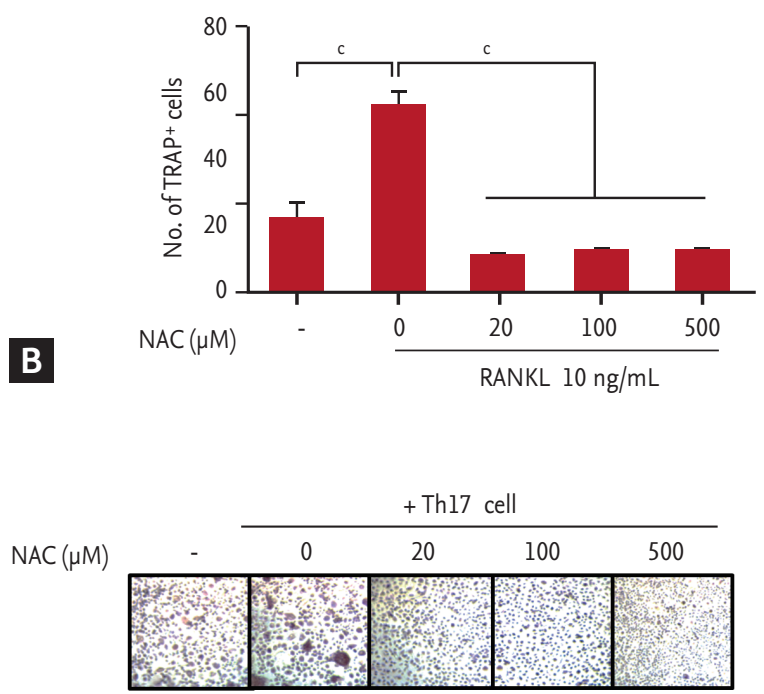

D

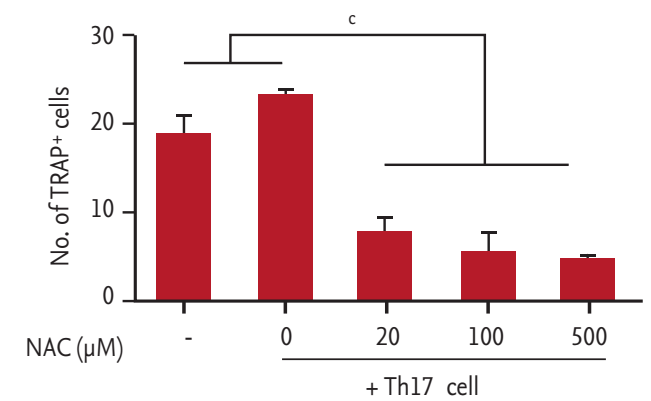

Figure 3. The effect of N-acetyl-1-cysteine (NAC) in osteoclast differentiation from peripheral blood monocytes. CDi4 ${ }^{+}$monocytes which were isolated from peripheral blood of healthy donor were pretreated with NAC for 3 hours, and then cultured with $25 \mathrm{ng} / \mathrm{mL}$ of macrophage colony-stimulating factor (M-CSF), and (A) $20 \mathrm{ng} / \mathrm{mL}$ of interleukin 17 (IL-17) or (B) $10 \mathrm{ng} / \mathrm{mL}$ of receptor activator of nuclear factor $\mathrm{\kappa B}$ ligand (RANKL). (C) Rheumatoid arthritis synovial fibroblasts were pretreated with NAC for 3 hours, then cultured with $20 \mathrm{ng} / \mathrm{mL}$ IL-17 for 72 hours and then co-cultured with CD14 ${ }^{+}$monocytes from the peripheral blood in the presence of M-CSF. (D) $\mathrm{CD}_{4}{ }^{+} \mathrm{T}$ cells were pretreated with NAC for 3 hours, then cultured with Thi7 condition for 72 hours and then co-cultured with $\mathrm{CD}_{4}{ }^{+}$monocytes from the peripheral blood in the presence of M-CSF. The data represent the mean \pm SEM of three independent experiments. TRAP, tartrate-resistant acid phosphatase; FLS, fibroblast-like synoviocyte. ${ }^{a} p<0.05,{ }^{b} p<0.01,{ }^{c} p<0.001$.

involved in this process in RA [26]. When osteoclast precursors were cultured with RA-FLS, the cells differentiated into mature osteoclasts more efficiently than when the cells were cultured alone [27]. Synovial fibroblasts are the primary cells involved in RA; these cells have many receptors and produce various inflammatory mediators, facilitating cross-talk with other immune cells and cytokines.

NAC is a thiolic antioxidant produced by the body and serves as a precursor of glutathione synthesis [28]. The efficacy of NAC has been investigated in clinical trials and experimental models of various respiratory conditions, such as chronic obstructive pulmonary disease, cirrhosis, portal hypertension, and acetaminophen poisoning [29]. NAC decreases the cytogenetic damage produced by exposure to cigarette smoke, whereas the intravenous or intra-arterial application of NAC is protective against the side effects of chemotherapy [30]. Additionally, as an 
Th17 condition

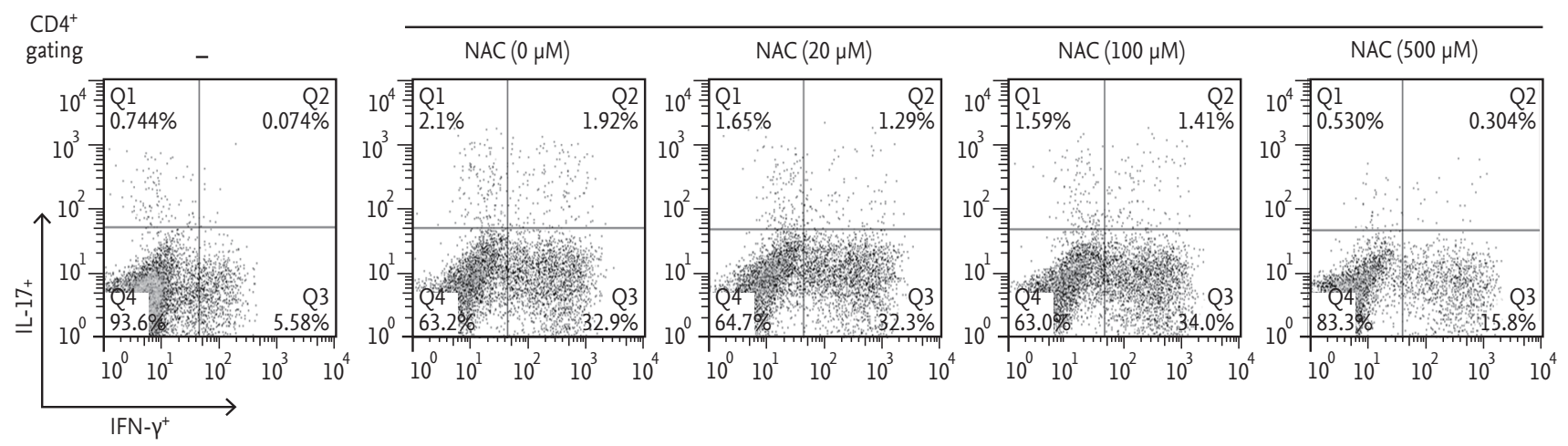

$\mathrm{CD}^{+}$

gating
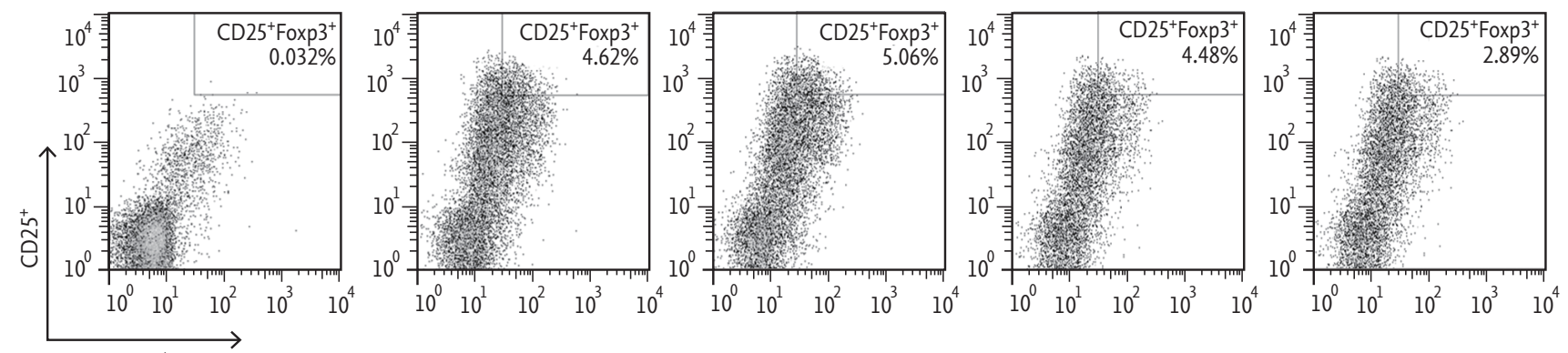

A
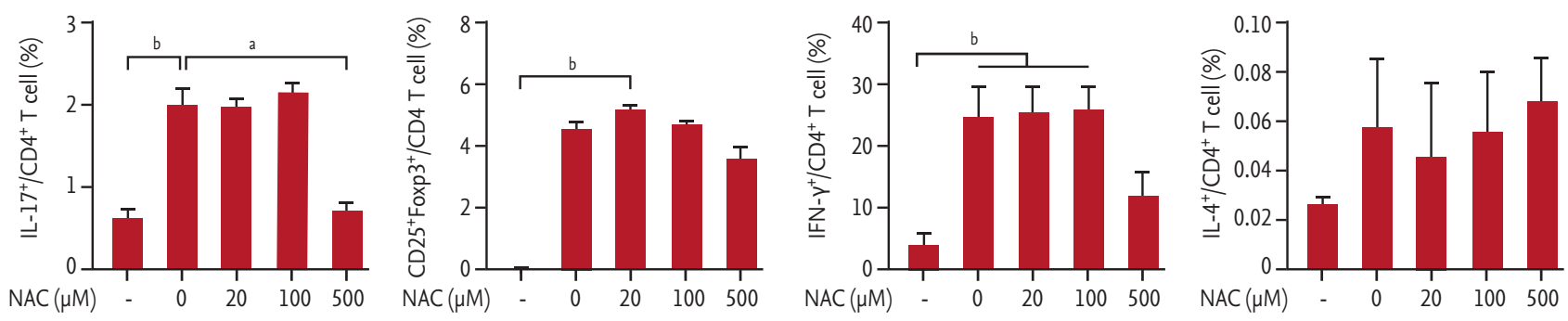

B
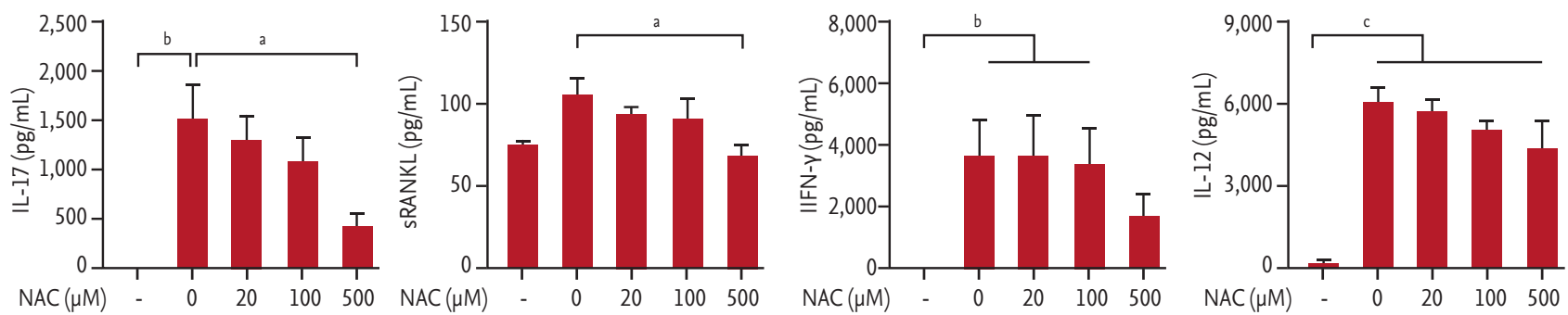

c

Figure 4. Effect of $\mathrm{N}$-acetyl-l-cysteine (NAC) on $\mathrm{CD}_{4}^{+} \mathrm{T}$ cells isolated from the peripheral blood mononuclear cells (PBMCs) of healthy donors and cultured under Th17-polarizing conditions. Human PBMCs $(\mathrm{n}=6)$ were isolated from healthy subjects and pre-incubated with with NAC $(20,100,500 \mu \mathrm{M})$ for 3 hours, and then cultured under Th17-polarizing conditions for 48 hours. (A) $\mathrm{CD}_{4}^{+} \mathrm{T}$ cells were gated for further analysis. Then, the percentage of $(\mathrm{B})$ interleukin $17^{+}\left(\mathrm{IL}_{-1} 7^{+}\right) / \mathrm{CD}_{4}{ }^{+} \mathrm{T} \mathrm{cells}, \mathrm{CD}_{25} 5^{+} \mathrm{FOXP}_{3}{ }^{+} /$ $\mathrm{CD}_{4}^{+} \mathrm{T}$ cells, interferon $\gamma^{+}\left(\mathrm{IFN}-\gamma^{+}\right) / \mathrm{CD}_{4}{ }^{+} \mathrm{T}$ cells, $\mathrm{IL}_{-} 4^{+} / \mathrm{CD}_{4}^{+} \mathrm{T}$ cells and was measured by flow cytometry. The production of (C) IL-17, soluble receptor activator of nuclear factor $\kappa-B$ ligand (sRANKL), IFN- $\gamma$, and IL-2 by Th17-polarizing CD $4{ }^{+} \mathrm{T}$ cells and secretion into the culture supernatant by enzyme-linked immunosorbent assay. Bars represent the mean \pm SEM. FOXP3, forkhead box $\mathrm{P}_{3} \cdot{ }^{\mathrm{a}} \mathrm{p}<0.05,{ }^{\mathrm{b}} \mathrm{p}<0.01,{ }^{\mathrm{c}} p<0.001$. 
antioxidant, NAC has inhibitory effects on osteoclastogenesis [12]. Accordingly, in this study, we investigated the antioxidant effects of NAC in IL-17-induced osteoclastogenesis and Th17 differentiation in RA.

We found that NAC reduced IL-17-induced RANKL gene expression and protein synthesis in RA synovial fibroblasts. IL-17 is a major cytokine involved in osteoclast differentiation. In our recent study, Th17 cytokines, such as IL-17, IL-21, and IL-22, were found to induce RANKL expression in RA synovial fibroblasts [23]. When peripheral blood monocytes are cultured with M-CSF and the Th17 cytokines in the absence of RANKL, the three Th17 cytokines significantly induce osteoclast differentiation from monocytes. Indeed, although IL-17 is a strong inducer of osteoclastogenesis, other Th17 cytokines, such as IL-21 and IL-22, also have osteoclastic effects, including effects that are different from those of IL-17. In RA treatment, the clinical efficacy and safety of anti-IL-17 monoclonal antibodies, such as secukinumab and ixekizumab, have been under clinical investigation [31]. However, in a phase II study of secukinumab for patients with RA, inadequate responses were observed. Despite this, phase III studies are still underway, and IL-17 is still promising target for RA treatment [32]. Anti-IL-17 monoclonal antibodies are developed by biologic methods; however, NAC is a chemical compound that has many advantages, including low cost, capacity for oral administration, short half-life, and low adverse effects. Similar benefits for the Janus kinase inhibitor tofacitinib have increased the use of this drug [33], although NAC would be more likely to have applications in enhancing the efficacy of other biologic therapies for preventing joint destruction.

In terms of signal transduction pathways, the inhibitory effects of NAC in IL-17-induced activation of RA synovial fibroblasts are known to be mediated through the mTOR/JNK/nuclear factor $\kappa \mathrm{B}(\mathrm{NF}-\kappa \mathrm{B})$ pathway. IL-17 activates mTOR, and NAC reduces IL-17-induced mTOR activation. Additionally, NAC activates AMPK, which functions to counteract the mTOR signal. In RA, inhibition of mTOR complex 1 (mTORC1) alters the ability of $\mathrm{IL} 17$ to induce mTORC1-dependent proliferation of RA synovial fibroblasts [34]. Interestingly, mTORC1 activity is increased in osteoclasts from patients with RA and in arthritic transgenic mice [35], and inhibition of mTOR results in downregulation of extracellular matrix diges- tive enzymes and induction of apoptosis in osteoclasts [35]. NAC inhibits nuclear translocation of NF- $\kappa B$ in RA synovial fibroblasts [36] and keratinocytes [37]. Moreover, Sakurada et al. [36] suggested that the inhibition of NF-кB nuclear translocation by NAC subsequently suppresses the induction of several cytokines and the expression of intracellular adhesion molecule-1.

In our study, we found that IL-17 directly induced osteoclast differentiation and indirectly induced osteoclastogenesis through co-culture of osteoclast precursors with RA synovial fibroblasts pre-exposed to IL-17 and Th17 cells. Additionally, NAC suppressed IL-17-induced osteoclastogenesis and osteoclast differentiation from co-cultures of their precursors with IL-17-pretreated synovial fibroblasts and Thr cells. Based on this result, IL-17 affects many stages of osteoclastogenesis, and NAC regulates IL-17-induced osteoclastogenesis.

Finally, we found that NAC regulated Th17 cell differentiation but did not affect the differentiation of regulator T cells or Th1 cells. This result suggested that NAC may modulate Thry cell differentiation and cytokine production; thereby, attenuating Th17 differentiation, which is crucial for the pathogenesis of synovial inflammation and bony destruction.

In conclusion, our results demonstrated that NAC regulated IL-17-induced RANKL expression in RA synovial fibroblasts and osteoclastogenesis from precursor cells. NAC also suppressed IL-17-activated synovial fibroblasts and Th17 cell-induced osteoclastogenesis. Although NAC has limitations in the control of inflammation and tissue damage, NAC could be administered as a supplemental therapeutic for prevention of inflammation and bone destruction in RA.

\section{KEY MESSAGE}

1. Stimulation of rheumatoid arthritis (RA) synovial fibroblasts with interleukin 17 (IL-17) promoted receptor activator of nuclear factor $\kappa-B$ ligand (RANKL) production.

2. N-acetyl-1-cysteine (NAC) blocked IL-17-induced RANKL production in a concentration-dependent manner.

3. NAC decreased IL-17-induced activation of mammalian target of rapamycin/c-Jun N-termi- 
nal kinase/inhibitor of $\kappa \mathrm{B}$ signaling.

4. Under Th17-polarizing conditions, NAC decreased Th17 cell differentiation.

5. NAC could be a supplementary therapeutic option for inflammatory processes in RA.

\section{Conflict of interest}

No potential conflict of interest relevant to this article was reported.

\section{Acknowledgments}

This research was supported by a grant of the Basic Science Research Program through the National Research Foundation of Korea (NRF) funded by the Ministry of Education, Science and Technology, Republic of Korea (NRF-2014R1A2A2A01007223) and the Korea Health Technology R\&D Project through the Korea Health Industry Development Institute (KHIDI), funded by the Ministry of Health to Welfare, Republic of Korea ( $\mathrm{HI}_{13} \mathrm{C}_{1704)}$.

\section{REFERENCES}

1. Harris ED Jr. Rheumatoid arthritis: pathophysiology and implications for therapy. N Engl J Med 1990;322:12771289.

2. Molenaar ET, Voskuyl AE, Dinant HJ, Bezemer PD, Boers M, Dijkmans BA. Progression of radiologic damage in patients with rheumatoid arthritis in clinical remission. Arthritis Rheum 2004;50:36-42.

3. Gravallese EM. Bone destruction in arthritis. Ann Rheum Dis 2002;61 Suppl 2:ii84-ii86.

4. Harrington LE, Hatton RD, Mangan PR, et al. Interleukin 17-producing $\mathrm{CD}_{4}+$ effector $\mathrm{T}$ cells develop via a lineage distinct from the $\mathrm{T}$ helper type 1 and 2 lineages. Nat Immunol 2005;6:1123-1132.

5. Tesmer LA, Lundy SK, Sarkar S, Fox DA. Th17 cells in human disease. Immunol Rev 2008;223:87-113.

6. Takayanagi H. Osteoimmunology: shared mechanisms and crosstalk between the immune and bone systems. Nat Rev Immunol 2007;7:292-304.

7. Jovanovic DV, Di Battista JA, Martel-Pelletier J, et al. IL17 stimulates the production and expression of proinflammatory cytokines, IL-beta and TNF-alpha, by human macrophages. J Immunol 1998;160:3513-3521.

8. Adamopoulos IE, Sabokbar A, Wordsworth BP, Carr A, Ferguson DJ, Athanasou NA. Synovial fluid macrophages are capable of osteoclast formation and resorption. J Pathol 2006;208:35-43.

9. Rosen CJ, Bouxsein ML. Mechanisms of disease: is osteoporosis the obesity of bone? Nat Clin Pract Rheumatol 2006;2:35-43.

10. Pfeilschifter J, Koditz R, Pfohl M, Schatz H. Changes in proinflammatory cytokine activity after menopause. Endocr Rev 2002;23:90-119.

11. Lean JM, Jagger CJ, Kirstein B, Fuller K, Chambers TJ. Hydrogen peroxide is essential for estrogen-deficiency bone loss and osteoclast formation. Endocrinology 2005;146:728-735.

12. Lee NK, Choi YG, Baik JY, et al. A crucial role for reactive oxygen species in RANKL-induced osteoclast differentiation. Blood 2005;106:852-859.

13. Lean JM, Davies JT, Fuller K, et al. A crucial role for thiol antioxidants in estrogen-deficiency bone loss. J Clin Invest 2003;112:915-923.

14. Chen JR, Lazarenko OP, Shankar K, Blackburn ML, Badger TM, Ronis MJ. A role for ethanol-induced oxidative stress in controlling lineage commitment of mesenchymal stromal cells through inhibition of Wnt/beta-catenin signaling. J Bone Miner Res 2010;25:1117-1127.

15. Hsu HY, Wen MH. Lipopolysaccharide-mediated reactive oxygen species and signal transduction in the regulation of interleukin-1 gene expression. J Biol Chem 2002;277:22131-22139.

16. Kim KW, Cho ML, Kim HR, et al. Up-regulation of stromal cell-derived factor 1 (CXCL12) production in rheumatoid synovial fibroblasts through interactions with T lymphocytes: role of interleukin-17 and CD4OL-CD40 interaction. Arthritis Rheum 2007;56:1076-1086.

17. Moon SJ, Park MK, Oh HJ, et al. Engagement of toll-like receptor 3 induces vascular endothelial growth factor and interleukin-8 in human rheumatoid synovial fibroblasts. Korean J Intern Med 2010;25:429-435.

18. Brentano F, Schorr O, Gay RE, Gay S, Kyburz D. RNA released from necrotic synovial fluid cells activates rheumatoid arthritis synovial fibroblasts via Toll-like receptor 3. Arthritis Rheum 2005;52:2656-2665.

19. Wu Y, Liu J, Feng X, et al. Synovial fibroblasts promote osteoclast formation by RANKL in a novel model of spontaneous erosive arthritis. Arthritis Rheum 2005;52:3257-3268. 
20. Kong YY, Yoshida H, Sarosi I, et al. OPGL is a key regulator of osteoclastogenesis, lymphocyte development and lymph-node organogenesis. Nature 1999;397:315-323.

21. Yasuda H, Shima N, Nakagawa N, et al. Osteoclast differentiation factor is a ligand for osteoprotegerin/osteoclastogenesis-inhibitory factor and is identical to TRANCE/ RANKL. Proc Natl Acad Sci U S A 1998;95:3597-3602.

22. Fujikawa $Y$, Sabokbar A, Neale SD, Itonaga I, Torisu T, Athanasou NA. The effect of macrophage-colony stimulating factor and other humoral factors (interleukin-1, -3, -6 , and -11, tumor necrosis factor-alpha, and granulocyte macrophage-colony stimulating factor) on human osteoclast formation from circulating cells. Bone 2001;28:261267.

23. Kim KW, Kim HR, Kim BM, Cho ML, Lee SH. Th17 cytokines regulate osteoclastogenesis in rheumatoid arthritis. Am J Pathol 2015;185:3011-3024.

24. Garrett IR, Boyce BF, Oreffo RO, Bonewald L, Poser J, Mundy GR. Oxygen-derived free radicals stimulate osteoclastic bone resorption in rodent bone in vitro and in vivo. J Clin Invest 1990;85:632-639.

25. Nakamura I, Takahashi N, Jimi E, Udagawa N, Suda T. Regulation of osteoclast function. Mod Rheumatol 2012;22:167-177.

26. Gronthos S, Zannettino AC. The role of the chemokine CXCL12 in osteoclastogenesis. Trends Endocrinol Metab 2007;18:108-113.

27. Kim HR, Kim KW, Kim BM, Cho ML, Lee SH. The effect of vascular endothelial growth factor on osteoclastogenesis in rheumatoid arthritis. PLoS One 2015;10:e0124909.

28. Gokcimen A, Cim A, Tola HT, et al. Protective effect of $\mathrm{N}$-acetylcysteine, caffeic acid and vitamin $\mathrm{E}$ on doxorubicin hepatotoxicity. Hum Exp Toxicol 2007;26:519-525.

29. Zafarullah M, Li WQ, Sylvester J, Ahmad M. Molecular mechanisms of N-acetylcysteine actions. Cell Mol Life Sci 2003;60:6-20.
30. Dickey DT, Muldoon LL, Doolittle ND, Peterson DR, Kraemer DF, Neuwelt EA. Effect of N-acetylcysteine route of administration on chemoprotection against cisplatin-induced toxicity in rat models. Cancer Chemother Pharmacol 2008;62:235-241.

31. Koenders MI, van den Berg WB. Secukinumab for rheumatology: development and its potential place in therapy. Drug Des Devel Ther 2016;10:2069-2080.

32. Genovese MC, Durez P, Richards HB, et al. Efficacy and safety of secukinumab in patients with rheumatoid arthritis: a phase II, dose-finding, double-blind, randomised, placebo controlled study. Ann Rheum Dis 2013;72:863-869.

33. Song GG, Bae SC, Lee YH. Efficacy and safety of tofacitinib for active rheumatoid arthritis with an inadequate response to methotrexate or disease-modifying antirheumatic drugs: a meta-analysis of randomized controlled trials. Korean J Intern Med 2014;29:656-663.

34. Saxena A, Raychaudhuri SK, Raychaudhuri SP. Interleukin-17-induced proliferation of fibroblast-like synovial cells is mTOR dependent. Arthritis Rheum 2011;63:14651466.

35. Cejka D, Hayer S, Niederreiter B, et al. Mammalian target of rapamycin signaling is crucial for joint destruction in experimental arthritis and is activated in osteoclasts from patients with rheumatoid arthritis. Arthritis Rheum 2010;62:2294-2302.

36. Sakurada S, Kato T, Okamoto T. Induction of cytokines and ICAM-1 by proinflammatory cytokines in primary rheumatoid synovial fibroblasts and inhibition by $\mathrm{N}$-acetyl-L-cysteine and aspirin. Int Immunol 1996;8:1483-1493.

37. Saliou C, Kitazawa M, McLaughlin L, et al. Antioxidants modulate acute solar ultraviolet radiation-induced NF-kappa-B activation in a human keratinocyte cell line. Free Radic Biol Med 1999;26:174-183. 